\title{
New Electronically Black Neutron Detectors
}

\section{MASTER}

\author{
D. M. Drake \\ W. C. Feldman \\ C. Hurlbut*
}

\section{DISCLAIMER}

\begin{abstract}
This report was prepared as an account of work sponsored by an agency of the United States Government. Neither the United States Government nor any agency thereof, nor any of their employees, makes any warranty, express or implied, or assumes any legal liability or responsibility for the accuracy, completeness, or usefulness of any information, apparatus, product, or process disclosed, or represents that its use would not infringe prirately owned rights. Reference herein to any specific commercial product, process, or service by trade name, trademark, manufacturer, or otherwise does not necessarily constitute or imply its endorsement, recommendation, or favoring by the United States Government or any agency thereof. The views and opinions of authors expressed herein do not necessarily state or reflect those of the United States Government or any agency thereor.
\end{abstract}


NEW ELECTRONICALLY BLACK NEUTRON DETECTORS

by

D. M. Drake, W. C. Feldman, and C. Hur1but

\begin{abstract}
Th1s report describes two neutron detectors that can function in a continuous radiation background. Both detectors identify neutrons by recording a proton recoil pulse followed by a characteristic capture pulse. This peculiar signature indicates that the neutron has lost all its energy in the scintillator. Resolutions and efficiencles have been measured for both detectors.
\end{abstract}

\title{
I. INTRODUCTION
}

Two new counters designed at the Los Alamos National Laboratory measure energy spectra of fast neutron fluxes in the presence of continuous gama-ray and charged particle backgrounds. These counters were designed specifically to fly in space where the naturally occurring neutron flux is almost Independent of time and time-of-flight techniques usually used in laboratory experiments to determine neutron energies are not useful.

Both counters use a delayed-pulse technique in which a fast neutron produces a light pulse by colliding vith one or more hydrogen atoms of a plastic scintillator. After the neutrons moderate sufficiently, some are captured by another element added to the counter. This capture yields a 
characteristic signature which identifies the first pulse as caused by a neutron that lost all of its serisible energy in the scintillator.

These neutron detectors are electronically "black" since each pulse accepted indicates the incident neutron was absorbed. We compare these electronically black detectors to geometrically black detectors developed at Argonne National Laboratory. 1

Similar double-pulse techniques have been used in a variety of measurements, including neutrino detection, ${ }^{2}$ measurement of $\bar{v}$ from fissionable elements, ${ }^{3}$ and the identification of pions (the second pulse is due to the $\pi \rightarrow \mathbf{u}$ decay) in elastic pion-scattering experiments. ${ }^{, 4}$

II. Plastic SCINTILlatoR COMBINED With A ${ }^{3}$ He PROPORTIONAL COUNTER

The first detector is a variant of a counter flown on the oGo 6 spacecraft, ${ }^{5}$ and it consisted of a ${ }^{3}$ He gas proportional counter surrounded by a scintillating plastic annulus. Because of severe weight, power, and telenetry restrictions, the OGO 6 detector had to be sma11 and its $\overline{1} 11$ potential could not be realized.

Figure 1 is a schematic drawing of this type of neutron spectrometer. It was built at Los Alamos and flown aboard a Nike-Tomahawk rocket from Kauai in September 1983. The outer detector was a 16-cm-o.d., 6-cm-i.d., by 20-cm-long hollow cylinder of BC 412 viewed by eight RCA 70132D photomultiplier tubes. The inner detector was a ${ }^{3} \mathrm{He}$ gas proportional counter having a $5.4-\mathrm{cm}-\mathrm{diam}$ by 20-cm-long active volume and pressurized to 10 atmospheres.

The plastic scintillator is sensitive to many types of radiation that are present in near-earth orbit, i.e., high-energy protons, gamma rays, electrons as well as neutrons, whereas the ${ }^{3}$ He counter is sensitive mainly to thermalized neutrons or penetrating charged particles. A pulse in the plastic followed by a ${ }^{3} \mathrm{He}$ pulse after some slowing-down time was most likely caused by an $n-p$ recoil evert in which the neutron had lost all its energy. A subset of pulses occurring in the plastic scintillator during the rocket test was caused by penetrating charged particles and was readily distinguishable from the neutron pulses because of their very large (saturated) signals from the plastic scintillator. A timing diagram for operation in the double-pulse mode is shown

"Information supplied by N. W. Hill, Oak Ridge National Laboratory, Oak Ridge, Tennessee. 


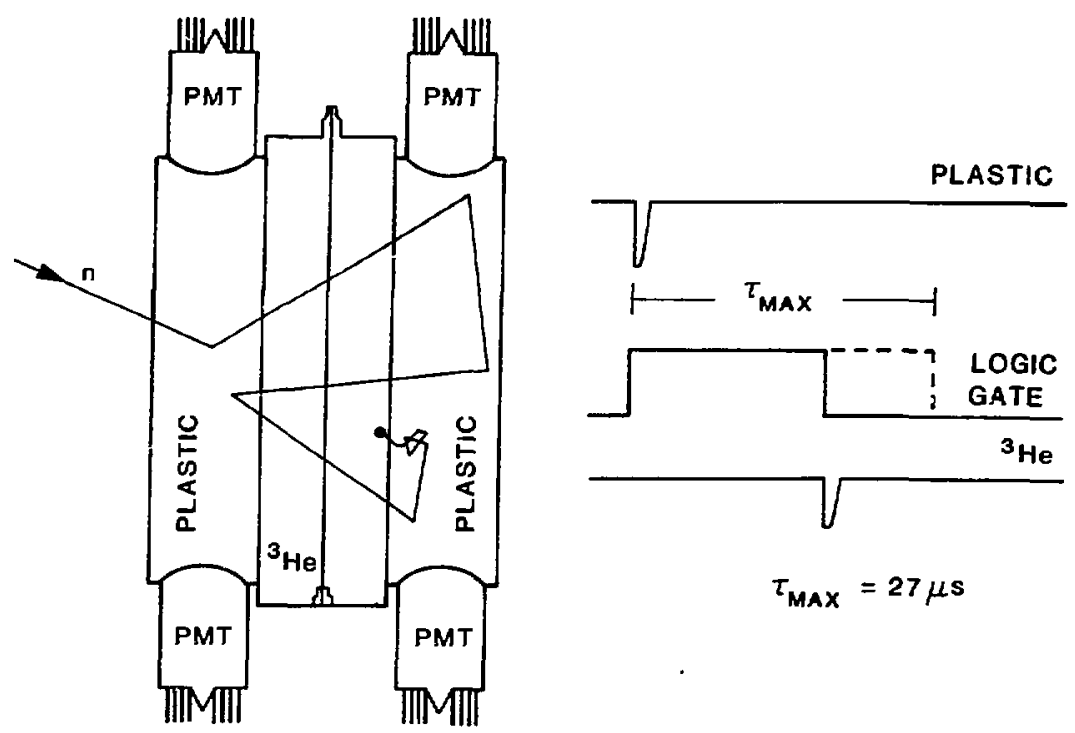

Fig. 1. Schematic drawing of the rocket neutron spectrometer showing the plastic-scintillator annulus surrounding the ${ }^{3}$ He proportional counter. A typical neutron interaction path is shown, starting with a scattering in the plastic and ending in the $3 \mathrm{He}+n+p+t$ in the proportional counter. On the right side of the figure, a time sequence for such an event is shown.

at the right in Fig. 1. The rocket instrument was designed to separately identify and record 1) events in the plastic scintillator that were unaccompanied by ${ }^{3} \mathrm{He}$ counter signals, 2) the total number of single ${ }^{3} \mathrm{He}$ counter events, and 3) plastic pulses accompanied by ${ }^{3}$ He pulses within 27 ss of the preceding plastic event.

The width of the time window was adjusted experimentaliy to accept $40 \%$ of all coincidences. Figure 2 shows the probability of a ${ }^{3}$ He event occurring within each $5 \neg$ s interval delayed a time, $\tau$, after an $n-p$ recoil event. An Am-B neutron source ${ }^{6}$ was used for this purpose. Inspection shows that relatively few coincidence events occurred at early time. This fact probably reflects the relatively long time required for low-energy, moderated neutrons to diffuse into the ${ }^{3}$ He counter. The vertical line at $\tau_{\max }=27 \mu \mathrm{s}$ Identifies the time interval within which $40 \%$ of all coincidence events occurred.

Figure 3 shows the absolute efficiency of coincidence events within $\tau_{\max }$ as a function of neutron energy in this mode of operation. It was measured usinj monoenergetic neutrons generated by the 6-MV Van de Graaff accelerator at Los Alamos. The axis of the spectroneter was positioned perpendicular to the 


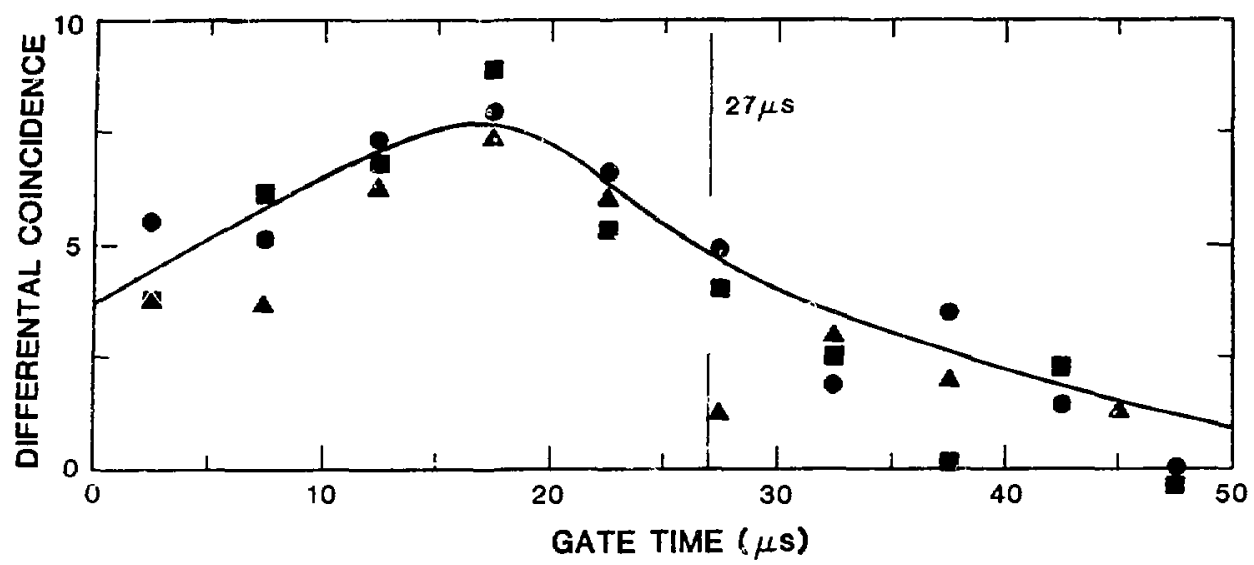

Fig. 2. Probability of double-pulse events plotted as a function of time. The 27- $\mu s$ window used during the rocket flight is shown. The different symbols refer to different series of measurements.

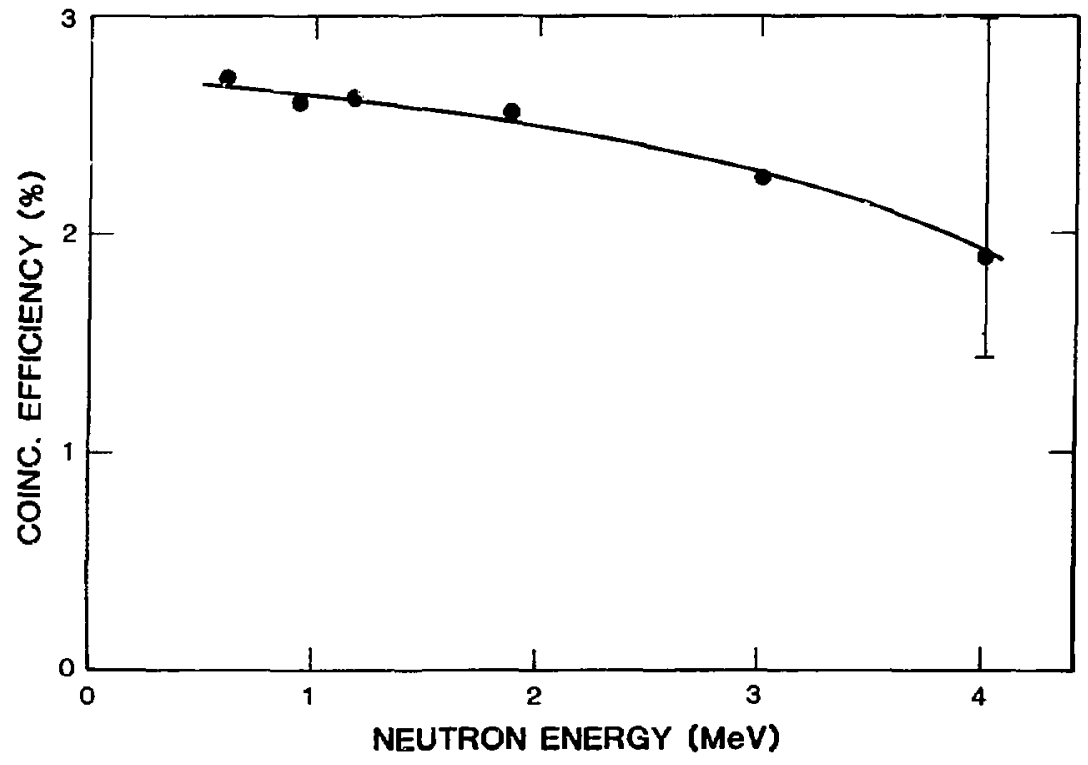

Fig. 3. Absolute neutron detection efficiency as a function of incident neutron energy. This efficiency is defined as the number of double-pulse events divided by the number of incident neutrons.

direction of the incident neutron beam for these measurements. The black detection efficiency is seen to decrease slowly from about $2.5 \%$ at $E=0.5 \mathrm{MeV}$ to about $2 \%$ at $E=4.0 \mathrm{MeV}$. However, the coincidence to singles fraction remained constant at 0.4 over this energy interval, as shown in Fig. 4 . Below 


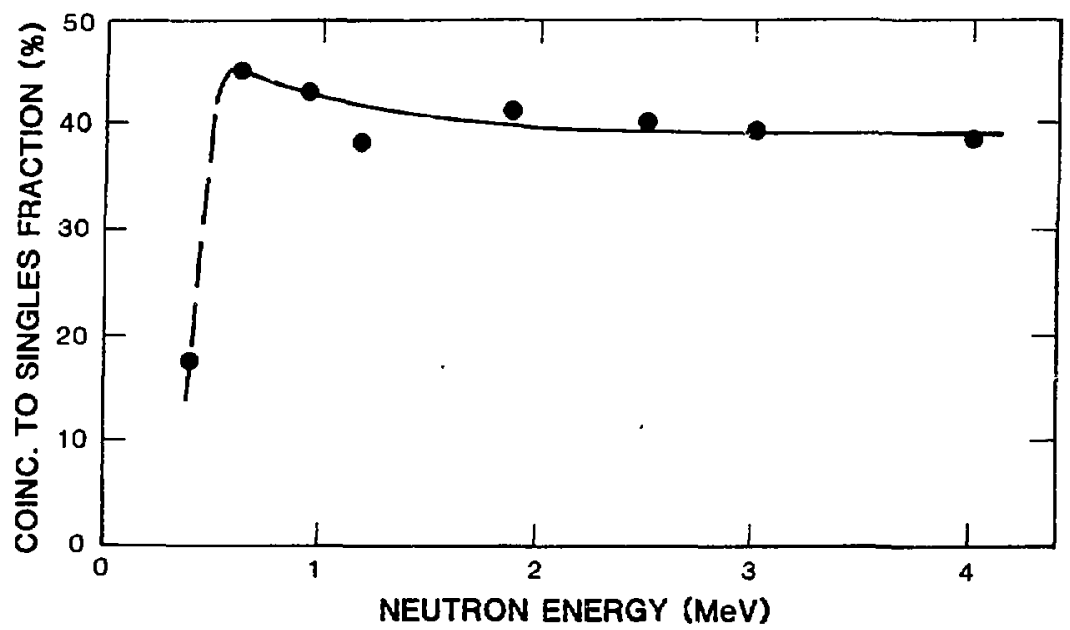

Fig. 4. A plot of the ratio (in percent) of the number of proton-recoil events giving pulses in coincidence wich a ${ }^{3}$ He counter pulse to the total number of proton-recoil events.

$E=450 \mathrm{keV}$, the efficiency for operation as a black detector dropped to unusably low values.

Figure 5 shows pulse-height spectra for monoenergetic neutrons between $0.6 \mathrm{MeV}$ and $3.0 \mathrm{MeV}$. They demonstrate operation as a black detector because they have near-Gaussian shapes with centroids, which move to higher pulse height (channel number) with increasing neutron energy. The centroid channel number as a function of neutron energy is plotted in Fig. 6. The nonlinear behavior at low energy is evident.

Because of the nonlinear light output of plastic scintillators, the widths of these pulse-height distributions depend on whether they are calculated directly from the detected light output or from the converted energy scale. However, all applications require resolution in energy rather than pulse height. All response spectra in Fig. 5 were therefore replotted using the conversion curve in Fig. 6 to obtain curves of counts versus neutron energy. The resultant response functions for $E=1.2 \mathrm{MeV}$ and $E=3.0 \mathrm{MeV}$ are seen in Fig. 7. The energy resolution specified as $\frac{\Delta E}{E}$ where $\Delta E$ is the full width at half maximum (FWHI) is seen to decrease with increasing neutron energy. Whereas $\Delta E / E \simeq 70 \%$ at $E=1.2 \mathrm{MeV}$, it reduces to $\sim 50 \%$ at $\mathrm{E}=3.0 \mathrm{MeV}$. The resolution of all spectra is summarized in Fig. 8 showing it is comparable to, but 


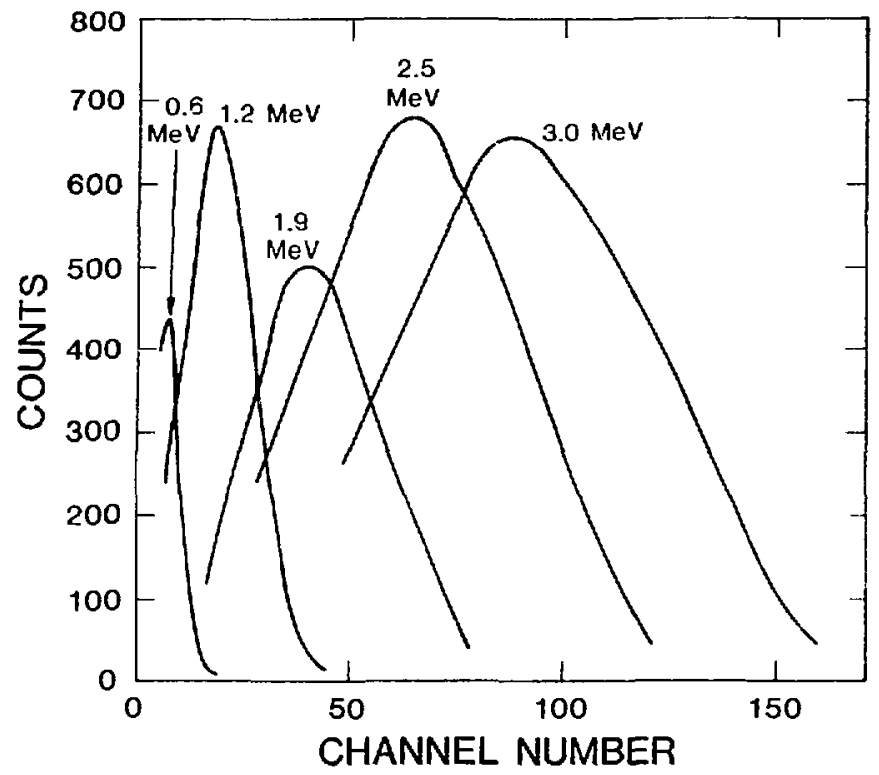

Fig. 5. Recoil pulse-height spectra for different incident neutron energies.

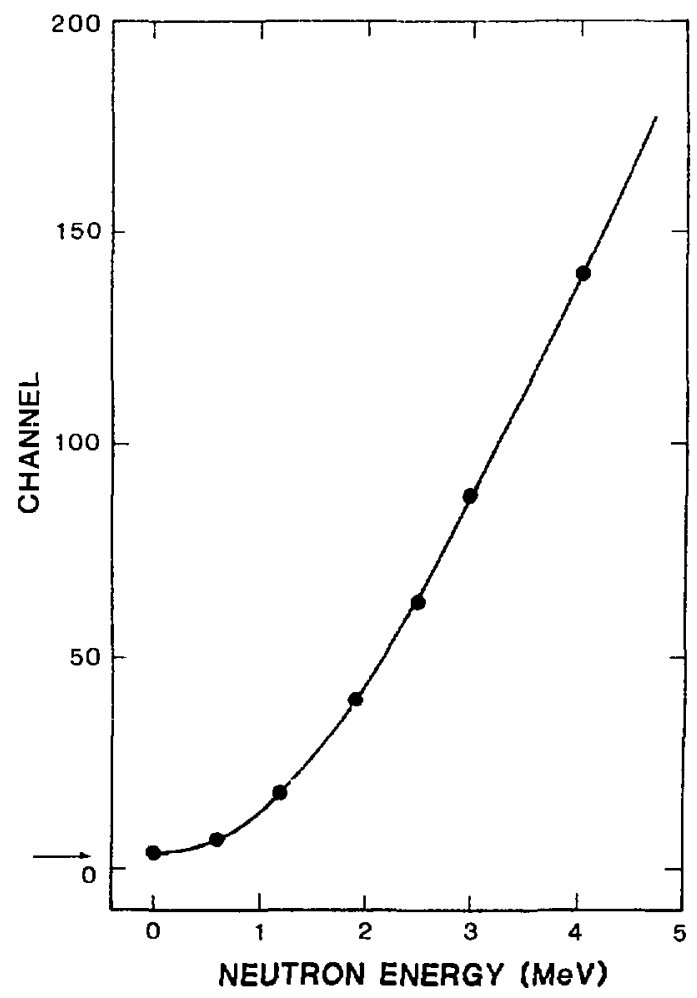

Fig. 6. Centroids of the distributions of Fig. 5 plotted as a function of incident neutron energy showing the nonlinear light output of the plastic scintillator. 


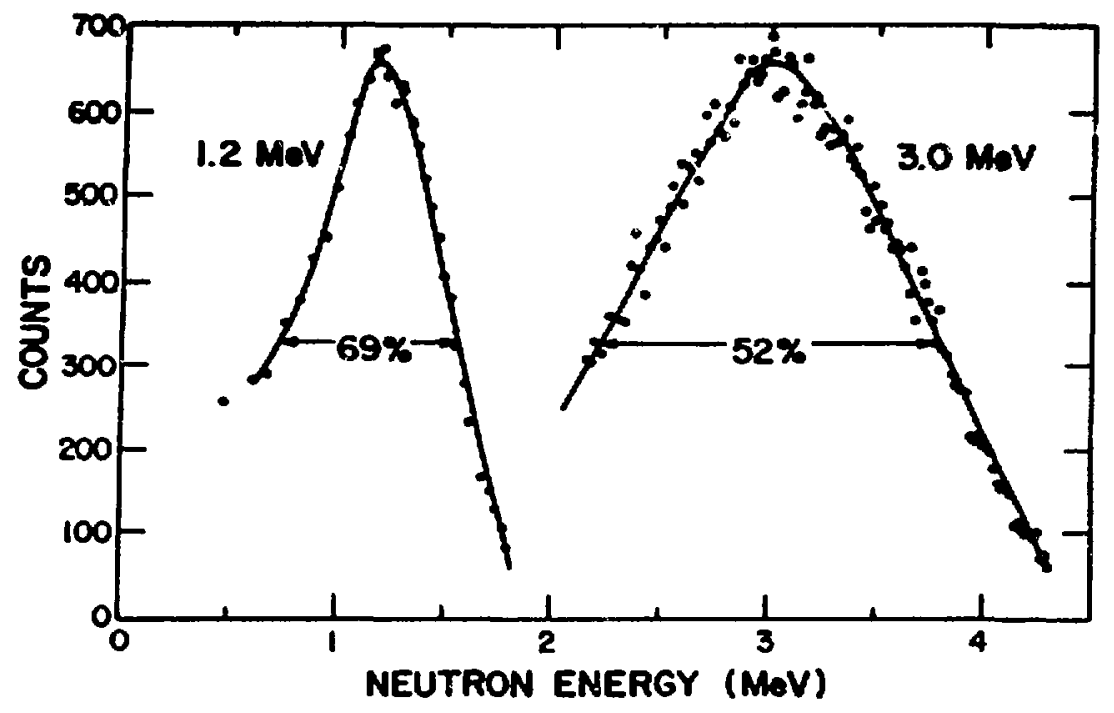

Fig. 7. Recoll spectra for 1.2 and $3.0-\mathrm{MeV}$ incldent neutrons plotted as a function of energy (rather than channel) so that resolution, $\Delta E / E$, can be obtained directly.

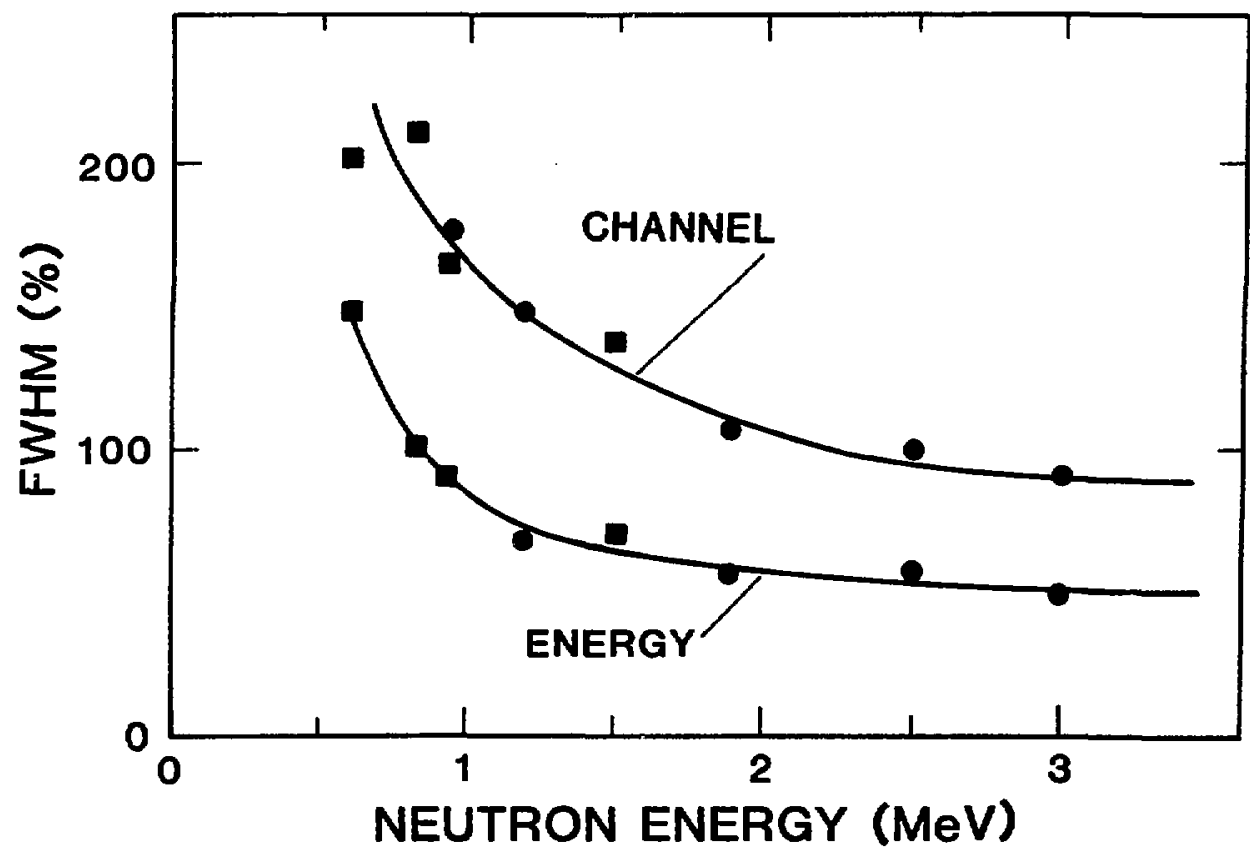

Fig. 8. Spectral resolution summarized as full widths at half maximum, FWHM, estimated both fron the measured pulse-height spectra (channel) and from the spectra replotted as a function of neutron energy using Fig. 6. The different symbols refer to different series of runs on the Van de Graaff. 
fractionally larger than, that achieved using the geometrically black detector developed at Argonne National Laboratory. 1 The disparity most 11kely reflects a different phototube-vlewing geometry and hence increased photon statistics in the Argonne detector. Additionally, most of the neutron interactions occurred In the same region of the Argonne detector, because it necessartly requires a collimated incldent beam, whereas interactions originate at any location in the electronically black detector.

\section{BORON-LOADED PLASTIC, BC 454}

The second counter described in this report is a boron-loaded plastic scintillator. Loading of scintillators with boron has been attempted before without significant success. ${ }^{7}$ The principle is similar to that of the

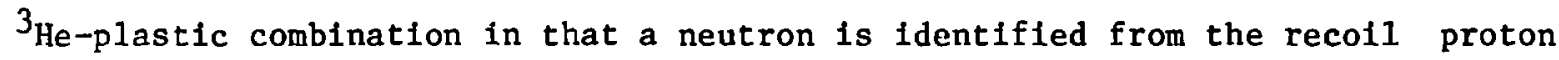
followed by a characteristic capture reaction, in this case ${ }^{10_{B}(n, \alpha Y)}{ }^{7} \mathrm{LI}$.

\section{A. Characteristics}

BC 454 is a new boron-loaded, plastic-scintillator material, marketed by Bicron Corp., in which some of the carbon atoms have been replaced in the molecular structure by the added boron. It is closely similar to $\mathrm{BC} 408$ having a density of $1.032 \mathrm{~g} / \mathrm{cm}^{3}$, a refractive index of 1.58 , and a wavelength at maximum emission of $425 \mathrm{~nm}$. Addition of boron in the form of a derivative uf decaborane followed by several stages of purification before copolymerization does not alter the gross physical characteristics of the final scintillator product. In particular, BC 454 appears as clear as BC 408 and displays the same blue shine when it is exposed to white light.

The light output of BC 454 decreases with increasing boron mass content as happened for the older boron-loaded, polystyrene-based plastic scintillator. ${ }^{7}$ The reduction relative to $B C 408$ ( $0 \%$ boron content) is shown in Fig. 9 as a function of boron content ranging from 0 to $15 \%$ by mass. It was measured using the Compton edge of ${ }^{137}$ Cs gamma-ray spectra from $2.5-\mathrm{cm}-\mathrm{d}$ lam by $2.5-\mathrm{cm}-1$ ong samples having $0,2,5,10$, and $15 \%$ boron mass content, respectively. 


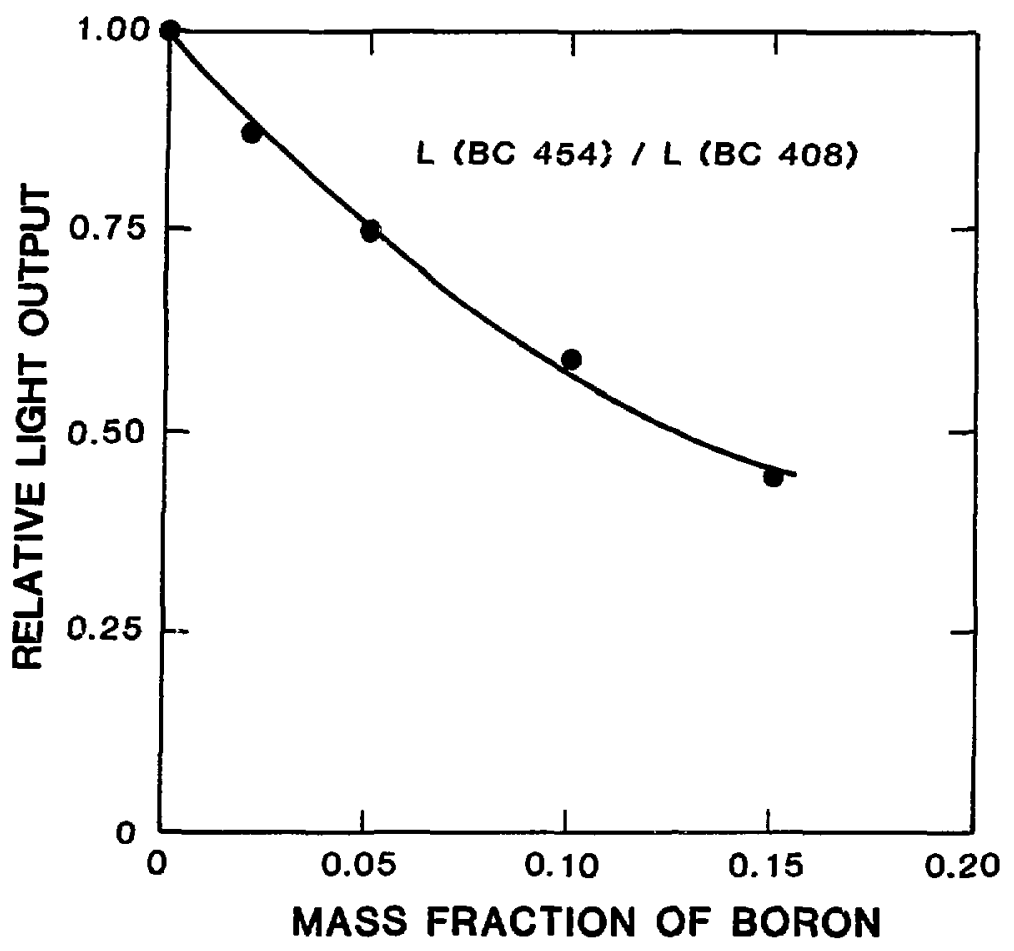

F1g. 9. Light output of $\mathrm{BC} 454$ relative to $\mathrm{BC} 408$ as a function of percent boron loading by weight.

B. Response of BC 454 to Fast Neutrons

The usual interaction of fast neutrons ( $\geq 200 \mathrm{keV}$ ) with the nuclei in BC 454 is by elastic or inelastic scattering. The energy deposited by the proton and/or carbon recolls results in a single pulse of light, which will be indistinguishable from that produced by elther a gamma ray or a penetrating charged particle. After each collision, the neutron can either escape or interact again with another nucleus in the scintillator. The probability that the scattering neutron will be captured by $10_{\mathrm{B}}$ can be computed by Monte Carlo techniques using known cross sections and the scintillator geometry.

The primary function served by the boron in BC 454 is to provide a detectable signal for low-energy neutrons. The cross section for ${ }^{10} \mathrm{~B}(\mathrm{n}, \alpha \gamma) 7_{\mathrm{LI}}$ closely follows a $1 / \mathrm{V}$ law for $E \leq 200 \mathrm{keV}$. The resulting absorption probability is therefore exponential with time constant, 
independent of neutron energy. Here $\mathrm{N}^{\left.10_{B}\right)}$ is the number density of $10_{\mathrm{B}}$ nuclei

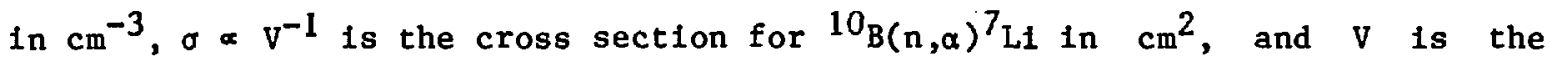
speed of the neutron in $\mathrm{cm} \mathrm{s}^{-1}$. Evaluating Eq. (1) for BC 454 having $5 \%$ mass content of natural boron gives $\tau(5 \%)=2.74 \mu \mathrm{s}$. Capture by other nuclei in BC 454 (5\%) accounts for less than $1 \%$ of the capture events.

The expected light output of $\mathrm{BC} 454$ resulting from capture by $10_{\mathrm{B}}$ can be calculated from the $Q$ value and kinematics of the reaction using Table 1 of Verbinski et al. 8 Assuming that captures produce a $478-\mathrm{keV}$ gamma ray, which does not interact in the scintillator, the $\alpha$ particle and ${ }^{7} \mathrm{~L} 1$ nucleus split the remaining $\sim 2.3 \mathrm{MeV}$ of the total $Q$ value in the ratio 7 to 4 . Interpolation of the data of Ref. 8 ylelds a minimum 11ght output equal to that produced by a 61-keV electron. Detection of both pulses provides an unambiguous identification of the incident radiation as a neutron and signals the fact that all its sensible energy was deposited in the scintillator. For neutron energles less than about $20 \mathrm{MeV}$, this occurs mostly in the form of proton recolls. Pulse-hefght analysis of the first pulse should then yield spectra close to those characteristic of black detectors.

\section{Callbration Tests}

A $7.6-\mathrm{cm}-\mathrm{diam}$ by $20-\mathrm{cm}-1$ ong cylinder of $5 \%$ BC 454 viewed by Hamamatsu R1652 photomultipliers on elther end was used for our initial tests of BC 454 with monoenergetic neutrons produced by the Los Alamos Van de Graff facility.

Figure 10 shows the pulse-height response of $\mathrm{BC} 454$ to low-energy neutrons through ${ }^{10} \mathrm{~B}(\mathrm{n}, \alpha \gamma)^{7} \mathrm{~L}$. The peak position corresponds to an electron of energy $76 \mathrm{keV}$, and the asymmetry is due to the occasional simultaneous detection of the 478-keV gamma ray.

Figures 11-13 show the pulse-height spectra of $0.5-, 3.0-$, and $5.7-\mathrm{MeV}$ neutrons. Because part of the electronic system was relatively slow, these spectra record the sum of the $n-p$ recoil pulses and the ${ }^{10} B(n, \alpha \gamma)^{7_{L 1}}$ pulses. This fact is most evident in Fig. 11 where the high-energy extension of the main peak corresponds to the Compton interaction of the 478-keV gamma ray resulting from ${ }^{10} \mathrm{~B}(\mathrm{n}, a \gamma)^{7} \mathrm{~L} 1$ with the $\mathrm{BC}$ 454. On the other hanc, the low-energy upturns of the main peaks in Figs, 12 and 13 result from room background 


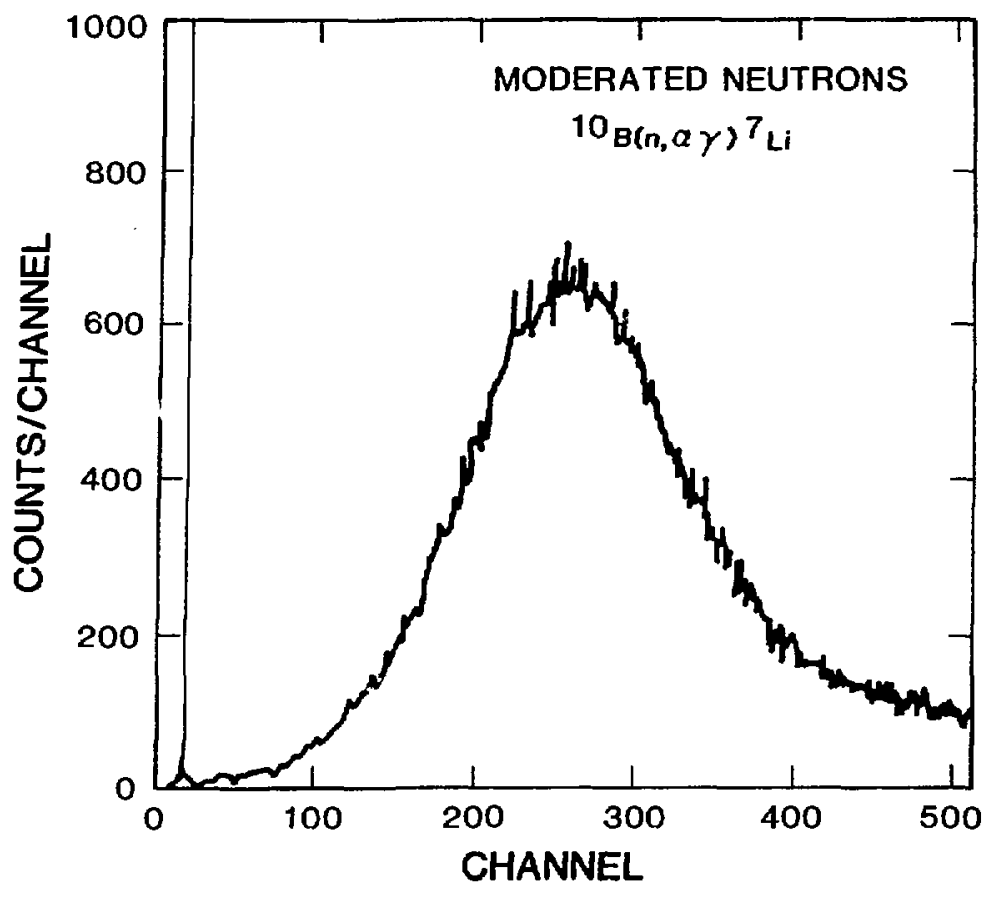

Fig. 10. A spectrum of the ${ }^{10} \mathrm{~B}(\mathrm{n}, \alpha \gamma)^{7} \mathrm{LI}$ reaction for low-energy neutrons in the $7.6-\mathrm{cm}$ diam $\times 20-\mathrm{cm}$ BC 454 scintillator. A few of the $0.47-\mathrm{MeV}$ gamma rays contribute to the detected light causing the asymmetry.

neutrons generated by interactions between the unfocused component of the deuterium beam anr surfaces of the beam line. Background runs with the deuterium target chamber evacuated verified that this background did not significantly interfere with the main peak. This peak then gives the pulse-height response of the $\mathrm{BC} 454$ to monoenergetic $3-\mathrm{MeV}$ and $5.7-\mathrm{MeV}$ neutrons in Figs. 12 and 13, respectively. The FWHM energy resolution of the 0.5, 3.0, and 5.7-MeV spectra are 0.55, 0.54, and 0.33, respectively, and, as with the rocket detector, are somewhat higher than the geometrically black detectors where comparable.

Figure 14 shows the exponential time dependence of the capture probability for incident neutrons of $0.5 \mathrm{MeV}$. The time constant for this curve is $2.7 \mu \mathrm{s}$ in agreement with the estimate of Eq. (1). After-pulsing in the photomultipliers caused occasional false coinctdences and is responsible for the discontinuity at early times. 


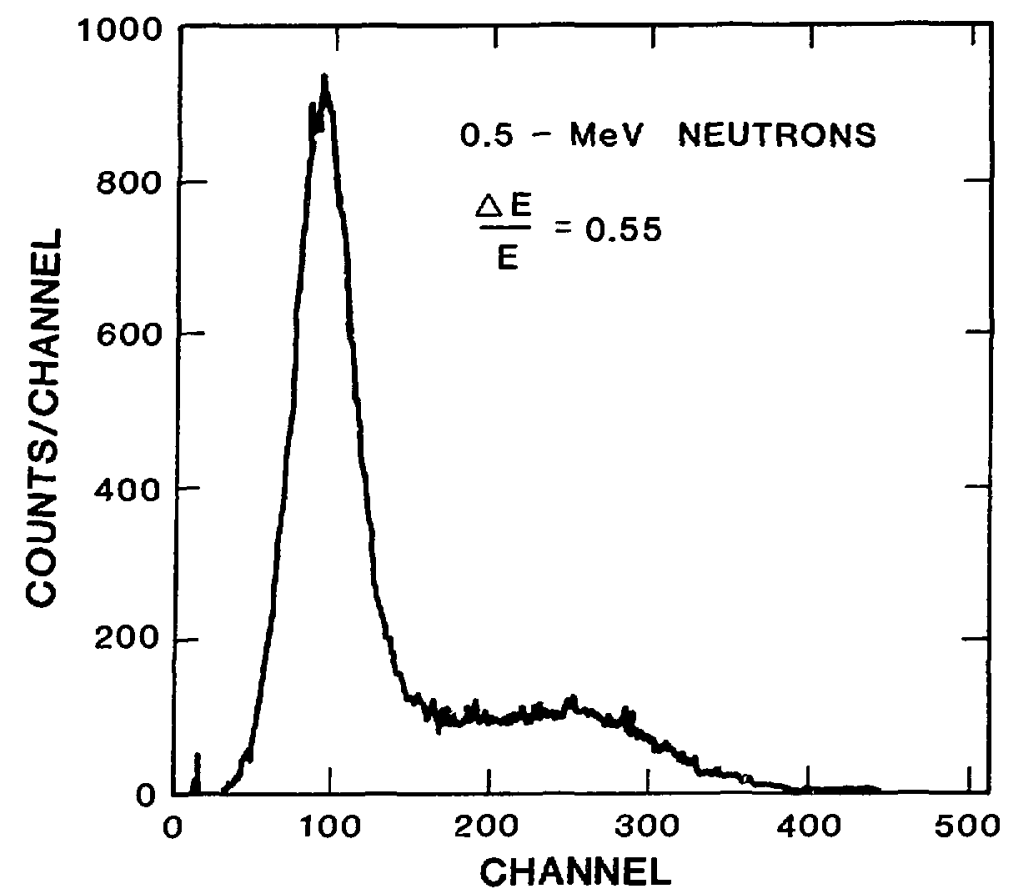

Fig. 11. Pulse-height spectrum for $0.5-\mathrm{MeV}$ neutrons incident on a $7.6-\mathrm{cm}$ diam $\times 20-\mathrm{cm}$ BC 454 cylinder.

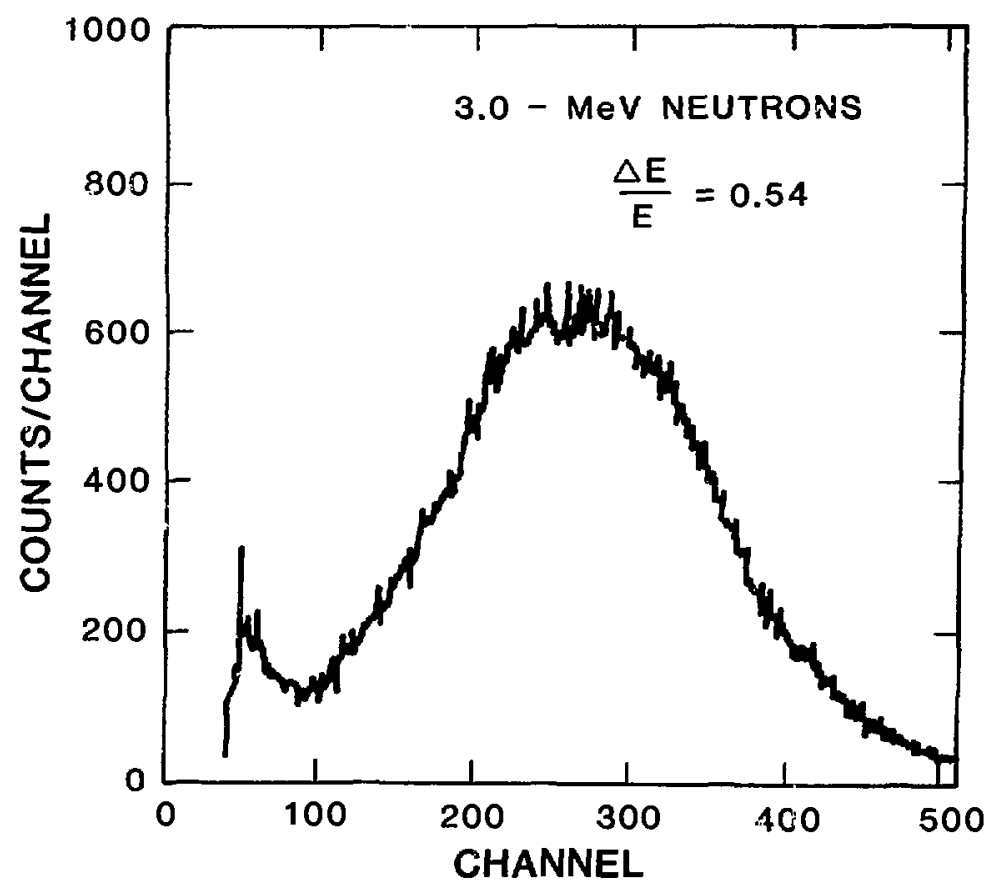

Fig. 12. Pulse-height spectrum for $3.0-\mathrm{MeV}$ neutrons incident on a $7.6-\mathrm{cm}$ diam $\times$ 20-cm BC 454 cylinder. 


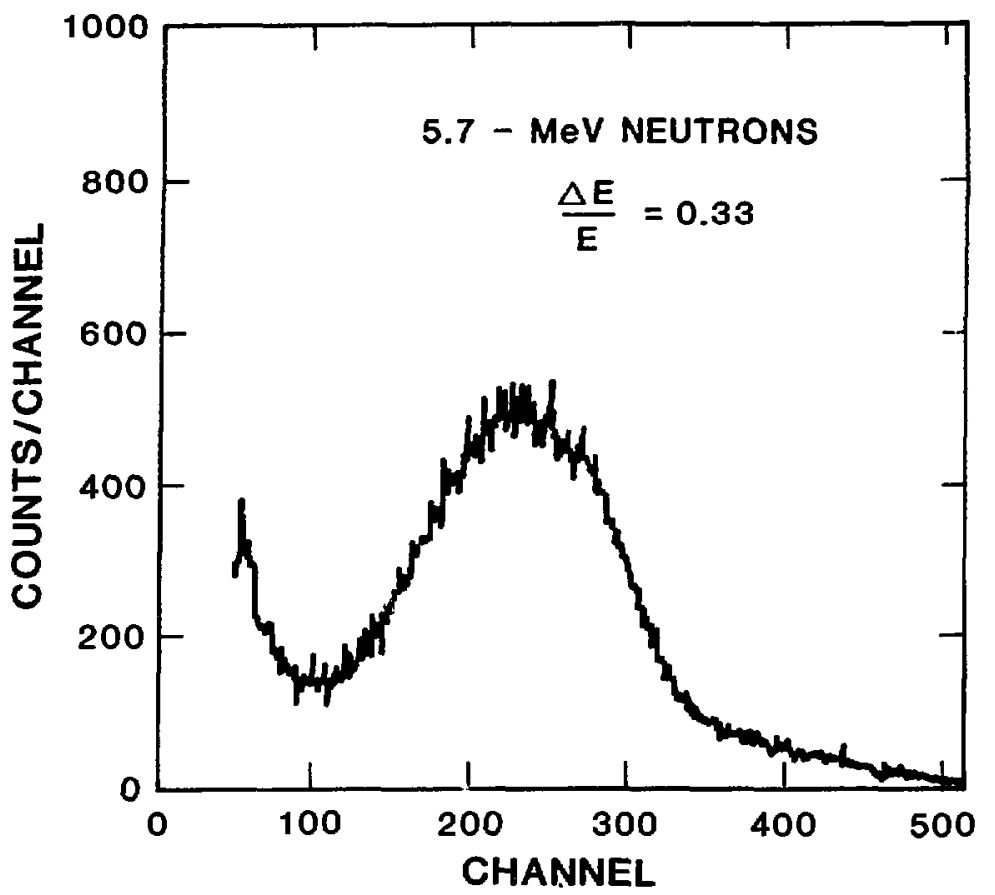

Fig. 13. Pulse-height spectrum for 5.7-MeV neutrons incident on a 7.6-cm diam $\times 20-\mathrm{cm}$ BC 454 cylinder.

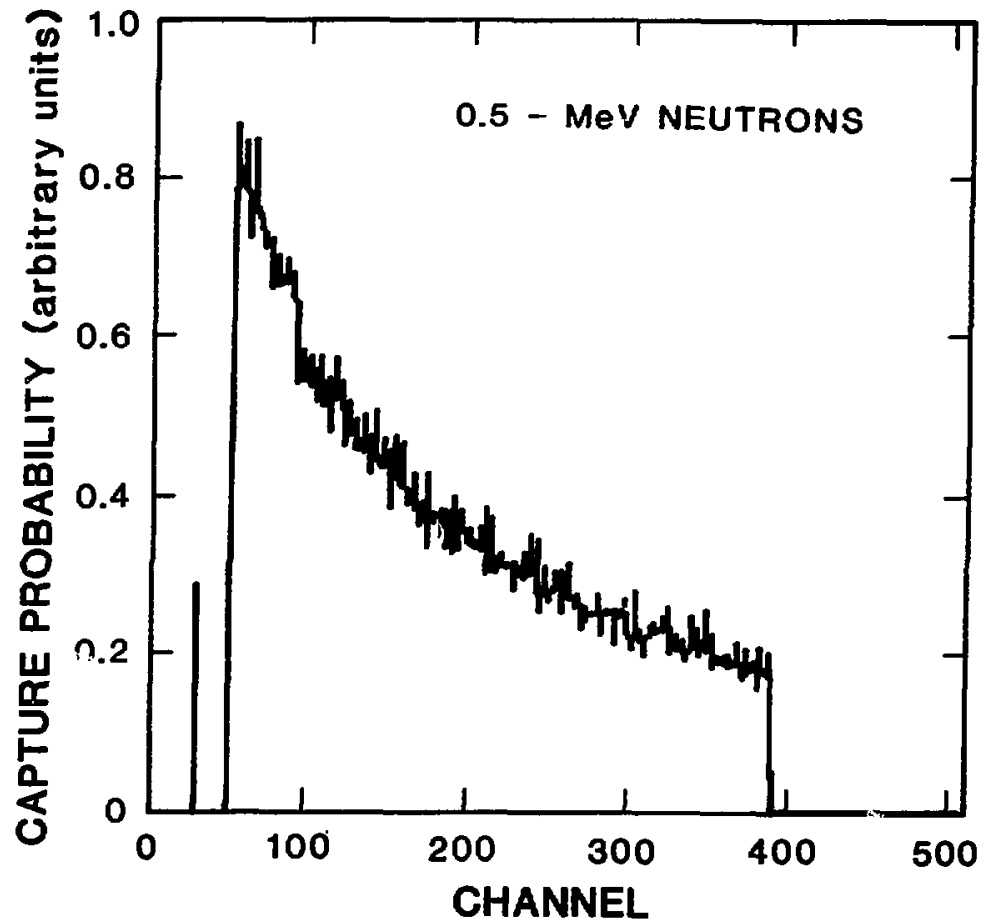

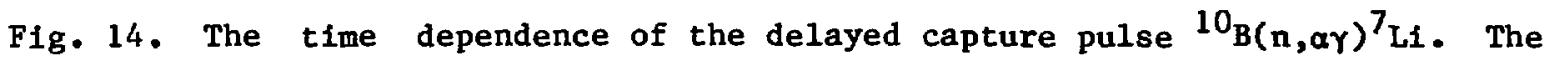
spectrum begins at about $200 \mathrm{~ns}$ and cuts off at $2.8 \mu \mathrm{s}$. The exponential decay is evident and the time constant is about $2.7 \mu \mathrm{s}$. 


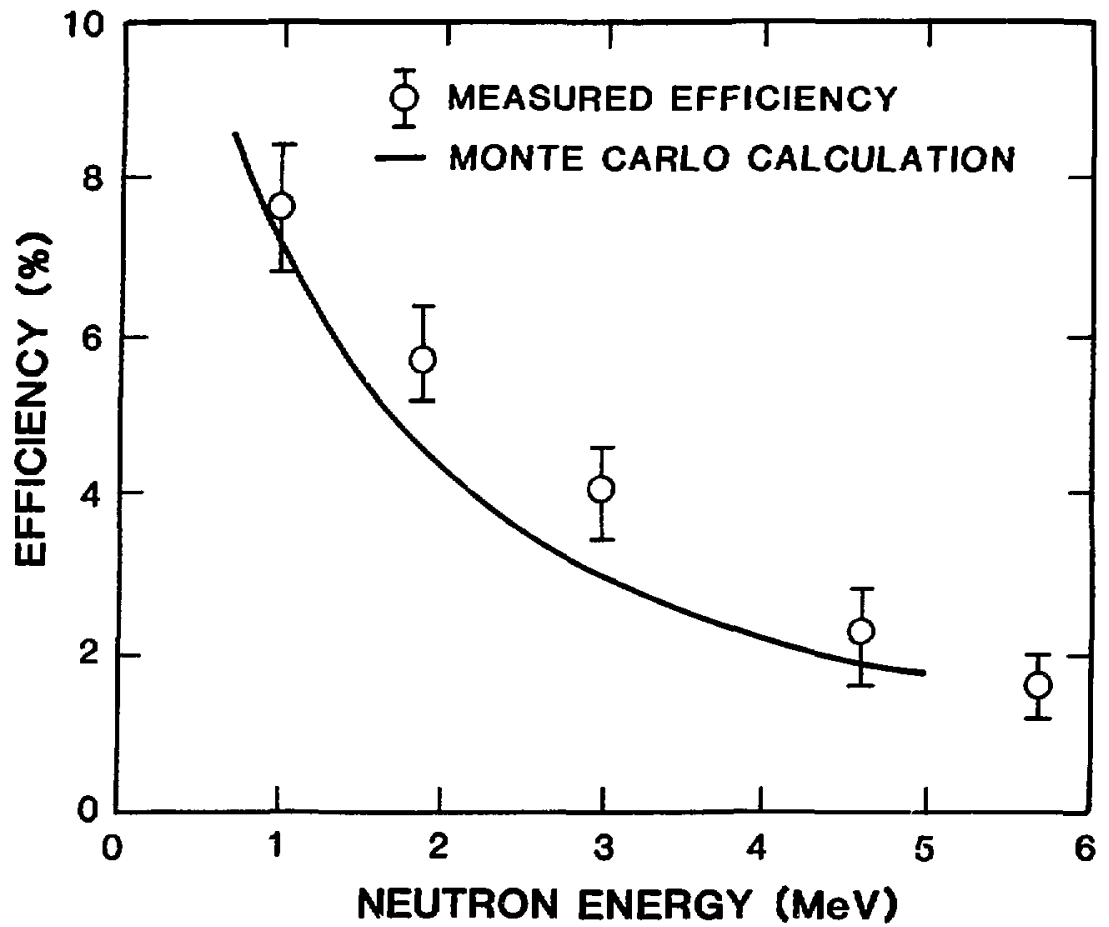

Fig. 15. Efficiency of the BC 454 electronlcally black detector as a function of incldent neutron energy. Also shown on this figure is an equivalent curve computed by a Monte Carlo technique.

The detection efficiency of a double-pulse coincidence was measured for neutron energies between 0.5 and $6 \mathrm{MeV}$ by normalizing to the absolute source neutron flux as measured using a thin-foil, proton-recoil detector. They are plotted in Fig. 15 along with efficiencies calculated using a Monte Carlo neutron transport code developed at Los Alamos. The agreement is reasonably good. Comparison with the efficiency curve given in Fig. 3 shows that the single BC 454 rod has a higher detection efficiency for energies in the range $0.5 \mathrm{MeV}<\mathrm{E}<4 \mathrm{MeV}$ than does the rocket neutron detector.

\section{SUMMARY}

The single rod of BC 454 tested at Los Alamos outperforms previous electronically black neutron detectors in two 1mportant ways. First, it provides a detection efficlency everywhere larger than that obtained prevlously, yet it has only about one quarter of the mass. Second, it requires a coincidence gate 10 times shorter than its predecessor based on a combination plastic scintillator and ${ }^{3}$ He gas proportional counter. The single BC 454 rod 
is therefore 10 times less susceptible to chance coincidences and can thus operate successfully in a higher background environment. Another important conclusion from our tests is that the Monte Carlo code at Los Alamos can be used to simulate the response functions of various detector designs. Therefore Monte Carlo can be used both to optimize prospective designs and to determine the full energy-angle response functions.

The resolution of either counters described here is comparatie to that of geometrically black detectors. Comparison of the few percent efficiencies of our detectors to the $\sim 100 \%$ quoted for geometrically black detectors is misleading because our detectors accept neutrons from any direction and do not require massive shielding to collimate the incident neutron beam. For space applications, a more reasonable figure of merit might be efficiency divided by spectrometer weight.

\section{ACKNOWLEDGMENTS}

We are grateful to J. Cessna for the electronic design of the rocket spectrometer, to R. C. Anderson for computer support, and to W. Moser for his inspired perseverance in the chemical laboratory work at Bicron.

\section{REFERENCES}

1. W. P. Poenitz, Argonne Nationaly Laboratory report ANL-7915 (1972) and Michael $M$. Meier, Proceedings of the International Specialists Symposium on Neutron Standards and Applicacions (Gaithersberg, Maryland, March 1977).

2. F. Reines and C. L. Cowan, Jr., Phys. Rev. 90, 492 (1953).

3. B. C. Diven, H. C. Martin, R. F. Taschek, and J. Terre11, Phys. Rev. 101, 1012 (1956).

4. D. J. Malbrough, R. D. Edge, C. W. Darden, T. Marks, B. M. Preedhome F. Bertrand, E. E. Gross, C. A. Ludemann, M. J. Saltmarsh, R. L. Burman, R. P. Redwine, M. A. Moinester, and K. Gotow, Phys. Rev. C 17, 1395 (1978).

5. J. A. Lockwood, E. L. Chupp, and R. W. Jenkins, IEEE Tranractions on Geoscience Electronics GE7 88 (1969).

6. Edgar A. Lorch, Int. J. of App1. Radiat. and Isot. 24, 585 (1973).

7. G. I. Anisimova, L. S. Danelyan, A. F. Zhigach, V. R. Lazarenko, V. N. Siryatskaya, and P.Z. Sorokin, Pri. Tekh. Eksp. 49 (1969). 
8. V. V. Verbinski, W. R. Burrus, T. A. Love, W. Zobel, N. W. Hill, and R. Textor, Nucl. Instrum. and Methods 65, 8 (1968). 